\title{
MAXIMAL FUNCTIONS OF MULTILINEAR MULTIPLIERS
}

\author{
Petr Honzík
}

AbStract. Let $m_{j}$ be Fourier multipliers on $\mathbb{R}^{2 d}$ that satisfy

$$
\left|\partial^{\alpha} m_{j}\left(\xi_{1}, \xi_{2}\right)\right| \leq A_{\alpha}\left(\left|\xi_{1}\right|+\left|\xi_{2}\right|\right)^{-|\alpha|}
$$

for sufficiently large $\alpha$ uniformly in $j$, for $j=1,2, \ldots, N$. We study the maximal operator of two variables

$$
\mathfrak{M}(f, g)(x)=\sup _{1 \leq j \leq N}\left|T_{m_{j}}(f, g)(x)\right|,
$$

where $T_{m_{j}}$ are the associated bilinear operators

$$
T_{m_{j}}(f, g)(x)=\int_{\mathbb{R}^{2 d}} m\left(\xi_{1}, \xi_{2}\right) \widehat{f}\left(\xi_{1}\right) \widehat{g}\left(\xi_{2}\right) e^{2 \pi i\left(\xi_{1}+\xi_{2}\right) \cdot x} \mathrm{~d} \xi_{1} \mathrm{~d} \xi_{2} .
$$

We prove that $\mathfrak{M}$ maps $L^{p_{1}}\left(\mathbb{R}^{d}\right) \times L^{p_{2}}\left(\mathbb{R}^{d}\right)$ to $L^{p}\left(\mathbb{R}^{d}\right)$ with norm at most a constant multiple $\sqrt{\log (N+2)}$. We also provide an example to indicate the sharpness of this result.

\section{Introduction}

A bilinear Fourier multiplier with symbol $m$ is a bilinear operator $T_{m}$ defined for functions $f, g$ in the Schwartz space $\mathcal{S}\left(\mathbb{R}^{d}\right)$ as follows

$$
T_{m}(f, g)(x)=\int_{\mathbb{R}^{2 d}} m\left(\xi_{1}, \xi_{2}\right) \widehat{f}\left(\xi_{1}\right) \widehat{g}\left(\xi_{2}\right) e^{2 \pi i\left(\xi_{1}+\xi_{2}\right) \cdot x} \mathrm{~d} \xi_{1} \mathrm{~d} \xi_{2} .
$$

Coifman and Meyer [5] proved that a bilinear multiplier operator $T_{m}$ is bounded from $L^{p_{1}}\left(\mathbb{R}^{d}\right) \times L^{p_{2}}\left(\mathbb{R}^{d}\right) \mapsto L^{p}\left(\mathbb{R}^{d}\right)$ whenever

$$
\left|\partial^{\alpha} m\left(\xi_{1}, \xi_{2}\right)\right| \leq A_{\alpha}\left(\left|\xi_{1}\right|+\left|\xi_{2}\right|\right)^{-|\alpha|}
$$

for sufficiently large multiindices $\alpha$ and

$$
\frac{1}{p_{1}}+\frac{1}{p_{2}}=\frac{1}{p}
$$

for $p_{1}, p_{2}, p \in(1, \infty)$. The range of $p$ was later extended to $(1 / 2, \infty)$ by Kenig and Stein [12] and independently by Grafakos and Torres [10].

In this article, we are going to study maximal operators associated with such bilinear multipliers. Suppose that we are given a family of bilinear symbols $\left\{m_{j}\right\}$, $j=1,2, \ldots, N$ that satisfy condition (1.1) uniformly in $j$. We consider the maximal operator

$$
\mathfrak{M}(f, g)(x)=\sup _{j}\left|T_{m_{j}}(f, g)(x)\right|
$$

and we are interested in its boundedness from $L^{p_{1}} \times L^{p_{2}} \mapsto L^{p}$ with norm as small as possible in terms of $N$.

Received by the editors March 28, 2005. 
The corresponding linear problem has been studied. Recall that a function $b$ on $\mathbb{R}^{d}$ is called a Mikhlin-Hörmander multiplier if it satisfies $\left|\partial^{\alpha} b(\xi)\right| \leq C_{\alpha}|\xi|^{-|\alpha|}$ for certain mutliindices $\alpha$. We refer to [13], [11], and [8] for properties of such multipliers. An example in [4] shows that a family of $N$ Mikhlin-Hörmander multipliers on $\mathbb{R}^{d}$ that satisfy uniform estimates forms a maximal operator whose $L^{p}$ norm is at least $C \sqrt{\log (N+2)}$, thus establishing the sharpness of this growth. In [9], it is proved that this estimate is also an upper bound for the $L^{p}$ norm. The purpose of this article is to establish analogous results in the bilinear setting. These results can be carried through for $m$-linear operators for $m \geq 3$ by straightforward modification of the arguments presented at the expense of some cumbersome notation.

We now state our main result.

Theorem 1.1. Let $1<p_{1}, p_{2}<\infty$ and $1 / 2<p<\infty$ satisfy (1.2). Given a family of bilinear symbols $\left\{m_{j}\right\}_{j=1}^{N}$ such that (1.1) holds for any multiindex $\alpha$ with constants $A_{\alpha}$ independent of $j$, the associated maximal operator $\mathfrak{M}$ satisfies an estimate

$$
\|\mathfrak{M}(f, g)\|_{p} \leq C \sqrt{\log (N+2)}\|f\|_{p_{1}}\|g\|_{p_{2}}
$$

for all functions $f \in L^{p_{1}}\left(\mathbb{R}^{d}\right)$ and $g \in L^{p_{2}}\left(\mathbb{R}^{d}\right)$. Conversely, for any $N \geq 1$ there is a family of symbols $m_{j}$ satisfying (1.1) uniformly and two Schwartz functions $f$ and $g$ such that

$$
\|\mathfrak{M}(f, g)\|_{p} \geq C \sqrt{\log (N+2)}\|f\|_{p_{1}}\|g\|_{p_{2}}
$$

The assumption of the theorem can be relaxed by assuming (1.1) only for multiindices $\alpha$ with $|\alpha|<K$, where $K$ is a constant which can be obtained from the proof. The constant depends on the dimension $d$ and is significantly larger than $d$.

\section{Notation and preliminaries}

We begin by introducing notation and some auxiliary operators and we remind the reader of some known results. We reserve the letter $C$ for any constant whose value may change. We are often going to stress the dependence of the constants on some parameters by using subscripts.

Given a locally integrable function $f$ we denote by $M f$ its Hardy-Littlewood maximal function, and for $r \geq 1$ we define $M_{r} f=\left(M|f|^{r}\right)^{1 / r}$ for functions in $L_{l o c}^{r}$. Jensen's inequality yields $M_{s} f(x) \leq M_{r} f(x)$ for any $x$ and $s \leq r$. Thus we have $M_{s} M_{r} f(x) \leq\left(M M|f|^{t}\right)^{1 / t}$ when $t=\max \{r, s\}$.

Our arguments depend on the Littlewood-Paley decomposition. We take a smooth function $\phi$, supported in $[1 / 2,2]$ with the property that $\sum_{i \in \mathbb{Z}} \phi\left(2^{-i} t\right)=1$ whenever $t \neq 0$. Denoting by $\widehat{h}$ the Fourier transform of a function $h$ and by $\mathcal{F}^{-1} h$ its inverse Fourier transform, we define the Littlewood-Paley operator

$$
\Delta_{i} f=\mathcal{F}^{-1}\left(\phi\left(2^{-i}|\cdot|\right) \widehat{f}\right), \quad i \in \mathbb{Z} .
$$

We recall that the vector valued operator $f \mapsto\left\{\Delta_{i} f\right\}_{i \in \mathbb{Z}}$ is bounded from $L^{p}$ to $L^{p}\left(l^{2}\right)$ for any $p>1$.

We shall use some elements of the martingale theory. We denote

$$
\mathcal{D}_{k}=\left\{\left[n_{1} 2^{-k},\left(n_{1}+1\right) 2^{-k}\right] \times \cdots \times\left[n_{d} 2^{-k},\left(n_{d}+1\right) 2^{-k}\right]: n_{i} \in \mathbb{Z}\right\}
$$


the family of dyadic cubes of side length $k$. We define the conditional expectation operator

$$
\mathbb{E}_{k} f(x)=2^{-k d} \sum_{Q \in \mathcal{D}_{k}} \chi_{Q}(x) \int_{Q} f(y) \mathrm{d} y
$$

and the martingale difference operator

$$
\mathbb{D}_{k} f(x)=\mathbb{E}_{k+1} f(x)-\mathbb{E}_{k} f(x) .
$$

The maximal martingale $\sup _{k}\left|\mathbb{E}_{k} f(x)\right|$ operator is pointwise bounded by $M f(x)$ and thus it is $L^{p}$ bounded. We also define the martingale square function

$$
S(f)=\left(\sum_{k \geq k_{0}}\left|\mathbb{D}_{k} f(x)\right|^{2}\right)^{1 / 2} .
$$

We use one sided martingales, so the constant $k_{0}$ specifies the starting level.

The standard method of handling multilinear multipliers is based on the observation that if the symbol $m$ si a tensor product $m\left(\xi_{1}, \xi_{2}\right)=a\left(\xi_{1}\right) b\left(\xi_{2}\right)$, then the operator $T_{m}$ splits as

$$
T_{m}(f, g)=T_{a}(f) T_{b}(g) .
$$

Therefore we will make use of the following tensor product lemma whose proof is more of less known (a sketch is included here for the reader's convenience.)

Lemma 2.1. Let $m$ be a function defined on $\mathbb{R}^{2 d}$ and supported inside $R=Q_{1} \times Q_{2}$, the product of two cubes $Q_{1}, Q_{2} \subset \mathbb{R}^{d}$, where $\operatorname{diam}\left(Q_{1}\right) \approx \operatorname{diam}\left(Q_{2}\right) \approx a$, which satisfies

$$
\left|\partial^{\alpha} m\left(\xi_{1}, \xi_{2}\right)\right| \leq A_{\alpha} a^{-|\alpha|}
$$

for any multiindex $\alpha$. Then we can find sequences of functions $a_{k_{1}}$ and $b_{k_{2}}$ and $a$ positive sequence $c^{k_{1}, k_{2}}$ such that

$$
\sum_{k_{1}, k_{2} \in \mathbb{Z}} c^{k_{1}, k_{2}} a_{k_{1}}\left(\xi_{1}\right) b_{k_{2}}\left(\xi_{2}\right)=m\left(\xi_{1}, \xi_{2}\right),
$$

for $|\alpha| \leq d$ we have

$$
\left|\partial^{\alpha} a_{k_{1}}\left(\xi_{1}\right)\right| \leq C_{\alpha}\left(1+\left|k_{1}\right|\right)^{|\alpha|} a^{-|\alpha|} \text { and }\left|\partial^{\alpha} b_{k_{2}}\left(\xi_{2}\right)\right| \leq C_{\alpha}\left(1+\left|k_{2}\right|\right)^{|\alpha|} a^{-|\alpha|}
$$

and such that for any $M>0$

$$
c^{k_{1}, k_{2}} \leq C_{M}\left(1+\left|k_{1}\right|+\left|k_{2}\right|\right)^{-M} .
$$

The functions $a_{k_{1}}\left(\xi_{1}\right)$ and $b_{k_{2}}\left(\xi_{2}\right)$ are supported inside $(1+\epsilon) Q_{1}$ and $(1+\epsilon) Q_{2}$, respectively, for some fixed $\epsilon>0$ independent of a.

Proof. By rescaling we can assume that $R$ is product of two unit cubes and

$$
\left|\partial^{\alpha} m\left(\xi_{1}, \xi_{2}\right)\right| \leq A_{\alpha} .
$$

The function $m$ can be represented as a two-dimensional Fourier series on $(1+\epsilon) R$ with faster than power decay. Each term of this series can then be written as a tensor product in $\xi_{1}$ and $\xi_{2}$. The functions $a_{k_{1}}$ and $b_{k_{2}}$ are then obtained as a smooth cutoffs adapted to $Q_{1}$ and $Q_{2}$. 
We note that if $a_{k_{1}}, b_{k_{2}}$ and $a$ are as above, one has

$$
\left\|\mathcal{F}^{-1}\left(a_{k_{1}}(a \cdot)\right)\right\|_{L^{1}} \leq C_{d}\left(1+\left|k_{1}\right|\right)^{d+1}
$$

and likewise for $\mathcal{F}^{-1}\left(b_{k_{2}}\right)$. Therefore the following estimates are valid

$$
\begin{aligned}
& \left|\mathcal{F}^{-1}\left(a_{k_{1}} \widehat{f}\right)\right|(x) \leq C\left(1+\left|k_{1}\right|\right)^{d+1}(M f)(x) \\
& \left|\mathcal{F}^{-1}\left(b_{k_{2}} \widehat{f}\right)\right|(x) \leq C\left(1+\left|k_{2}\right|\right)^{d+1}(M f)(x),
\end{aligned}
$$

with a constant $C$ depending only on the dimension and on the constants $A_{\alpha}$.

\section{Family with sharp growth}

In this section, we provide an example of a countable family of bilinear symbols $m_{j}$, such that condition (1.1) is valid and such that the maximal operator $\mathfrak{M}_{N}$ associated to first $N$ of them has norm bigger than $C \sqrt{\log N}$, with $C$ independent of $N$. For simplicity we take $d=1$ but we point out that a generalization for $d>1$ is straightforward. The method of the construction is similar to that in [4] but we need to take the second function into consideration.

We construct the symbols $m_{j}, j \geq 0$. Fix a smooth function $\psi$, supported in $[-1 / 4,1 / 4]$ such that for $|\xi| \leq 1 / 8$ we have $\psi(\xi)=1$. We denote by $j(k)$ the $k$-th digit of binary representation of $j$. Then, we put

$$
m_{j}\left(\xi_{1}, \xi_{2}\right)=\sum_{k=1}^{\infty} j(k) \psi\left(2^{-k} \xi_{2}\right) \psi\left(2^{-k} \xi_{1}-1\right) .
$$

It is obvious that these symbols satisfy condition (1.1) uniformly in $j$.

To construct the test function, we are going to use a smooth non-zero function $\phi$ with Fourier transform supported in $[-1 / 8,1 / 8]$. Let us take $l$ such that $2^{l} \leq N<$ $2^{l+1}$. We then put

$$
\widehat{f}(\xi)=\sum_{k=1}^{l} \widehat{\phi}\left(\xi-2^{k}\right) .
$$

With the aid of the Littlewood-Paley theory we see that $C\|f\|_{p_{1}} \leq l^{1 / 2}\|\phi\|_{p_{1}}$.

We are going to examine the norm of $\mathfrak{M}_{N}(f, \phi)$. Observe that

$$
T_{m_{j}}(f, \phi)(x)=\sum_{k=1}^{l} j(k) e^{2 \pi i 2^{k} x} \phi^{2}(x) .
$$

Given $x$, we now find an index $j, j \leq N$ such that $\left|\sum_{k=1}^{l} j(k) e^{2 \pi i 2^{k} x}\right| \geq l / 8$. This can be done by defining four index sets: $S_{0}, S_{1}, S_{2}$ and $S_{3}$, where

$$
S_{n}=\left\{1 \leq k \leq N: \operatorname{Re} e^{2 \pi i\left(2^{k} x+n \pi / 2\right)} \geq 1 / \sqrt{2}\right\} .
$$

Then we have $\cup_{i} S_{i}=\{1, \ldots, l\}$ and so we can select $n$ such that $\left|S_{n}\right| \geq N / 4$ and put $j(k)=\chi_{S_{n}}(k)$. By this selection of the index $j$ it follows that $\left|T_{m_{j}}(f, \phi)(x)\right| \geq$ $l \phi^{2}(x) / 8$ which gives

$$
\left\|\mathfrak{M}_{N}(f, \phi)\right\|_{p} \geq C l\left\|\phi^{2}\right\|_{p}
$$

We of course have

$$
\left\|\phi^{2}\right\|_{p} \geq C\|\phi\|_{p_{1}}\|\phi\|_{p_{2}}
$$


since $\|\phi\|_{p_{1}}$ and $\|\phi\|_{p_{2}}$ are both finite numbers and we can choose $C$ approprietly. Thus we get

$$
\left\|\mathfrak{M}_{N}(f, \phi)\right\|_{p} \geq C l^{1 / 2}\|f\|_{p_{1}}\|\phi\|_{p_{2}} \geq C(\log N)^{1 / 2}\|f\|_{p_{1}}\|\phi\|_{p_{2}} .
$$

\section{Decomposition of the symbols}

We shall now prove (1.3). The proof follows the same pattern as that of the classical (non-maximal) result.

First we decompose the symbol of each multiplier to the "diagonal" part and the "axial" part. We fix a large constant $K>0$ and denote

$$
\Gamma=\left\{\left(\xi_{1}, \xi_{2}\right): K^{-1}\left|\xi_{1}\right| \leq\left|\xi_{2}\right| \leq K\left|\xi_{1}\right|\right\}
$$

and

$$
\Gamma^{\prime}=\left\{\left(\xi_{1}, \xi_{2}\right): \frac{K^{-1}}{2}\left|\xi_{1}\right| \leq\left|\xi_{2}\right| \leq 2 K\left|\xi_{1}\right|\right\} .
$$

Consider smooth homogeneous partition of the unity $\psi_{1}(\xi)+\psi_{2}(\xi)=1$ for $\xi \neq 0$ such that $\psi_{1}$ is supported in $\mathbb{R}^{2 d} \backslash \Gamma$ and $\psi_{2}$ in $\Gamma^{\prime}$.

Thus, we shall have a decomposition $m_{j}=m_{j}^{1}+m_{j}^{2}=m_{j} \psi_{1}+m_{j} \psi_{2}$. Naturally, condition (1.1) is still valid for the new symbols with comparable constants (and thus uniformly in $j$ ). For admisible functions $f$ and $g$, we have a pointwise inequality $\mathfrak{M}(f, g) \leq \mathfrak{M}^{1}(f, g)+\mathfrak{M}^{2}(f, g)$, where the operator $\mathfrak{M}^{r}$ is associated with the family $m_{j}^{r}$. Furthermore, we denote

$$
\Omega=\left\{\xi=\left(\xi^{1}, \ldots, \xi^{d}\right) \in \mathbb{R}^{d}: \sum_{i=2}^{d}\left|\xi^{i}\right|^{2} \leq\left|\xi^{1}\right|^{2} ; \xi^{1}>0\right\}
$$

a cone in $\mathbb{R}^{d}$. By a simple decomposition and symmetry argument we can assume that symbols $m_{j}^{1}$ are supported in

$$
\left\{\left(\xi_{1}, \xi_{2}\right):\left|\xi_{2}\right| \leq K^{-1}\left|\xi_{1}\right|\right\} \cap \Omega^{2}
$$

and $m_{j}^{2}$ in $\Gamma^{\prime} \cap \Omega^{2}$

\section{The part $\mathfrak{M}^{2}$}

First, we control the operator $\mathfrak{M}^{2}$, which is in fact bounded uniformly in $N$. We set $m_{j, i}^{2}\left(\xi_{1}, \xi_{2}\right)=\phi\left(2^{-i}\left|\left(\xi_{1}, \xi_{2}\right)\right|\right) m_{j}^{2}\left(\xi_{1}, \xi_{2}\right)$. We observe that $\sum_{i} m_{j, i}^{2}\left(\xi_{1}, \xi_{2}\right)=m_{j}^{2}\left(\xi_{1}, \xi_{2}\right)$ and that each of the pieces $m_{j, i}^{2}$ is supported in an $\epsilon 2^{i}$-interior of some cube $Q$ which such that $\operatorname{diam}(Q) \leq C_{1} 2^{i}$, with $C_{1}$ independent of $i$ and for any $\left(\xi_{1}, \xi_{2}\right) \in Q$ we have $\left|\xi_{1}\right| \geq C_{2} 2^{i},\left|\xi_{2}\right| \geq C_{2} 2^{i}$, with $C_{2}>0$ independent of $i$.

Now we are in a position to apply Lemma 2.1, to decompose

$$
m_{j, i}^{2}\left(\xi_{1}, \xi_{2}\right)=\sum_{k_{1}, k_{2}} c_{i, j}^{k_{1}, k_{2}} a_{i, j}^{k_{1}}\left(\xi_{1}\right) b_{i, j}^{k_{2}}\left(\xi_{2}\right)
$$

such that

$$
\begin{aligned}
\left|\partial^{\alpha} a_{i, j}^{k_{1}}\left(\xi_{1}\right)\right| & \leq C\left(1+\left|k_{1}\right|\right)^{|\alpha|}\left|\xi_{1}\right|^{-|\alpha|} \quad \text { and } \\
\left|\partial^{\alpha} b_{i, j}^{k_{2}}\left(\xi_{2}\right)\right| & \leq C\left(1+\left|k_{2}\right|\right)^{|\alpha|}\left|\xi_{2}\right|^{-|\alpha|},
\end{aligned}
$$


the factors $c_{i, j}^{k_{1}, k_{2}}$ have fast decay and the functions $a_{i, j}^{k_{1}}$ and $b_{i, j}^{k_{2}}$ are supported in the cube $\left[C^{-1} 2^{-i}, C 2^{-i+1}\right]^{d}$. Thus, we can write

$$
\mathfrak{M}^{2}(f, g)(x)=\sup _{j}\left|\sum_{i, k_{1}, k_{2}} c_{i, j}^{k_{1}, k_{2}} \mathcal{F}^{-1}\left(a_{i, j}^{k_{1}} \widehat{f}\right)(x) \mathcal{F}^{-1}\left(b_{i, j}^{k_{2}} \widehat{g}\right)(x)\right| .
$$

We denote $c^{k_{1}, k_{2}}=\sup _{i, j} c_{i, j}^{k_{1}, k_{2}}$. The Cauchy-Schwarz inequality then yields the estimate

$$
\mathfrak{M}^{2}(f, g)(x) \leq \sup _{j} \sum_{k_{1}, k_{2}} c^{k_{1}, k_{2}}\left(\sum_{i}\left|\mathcal{F}^{-1}\left(a_{i, j}^{k_{1}} \widehat{f}\right)(x)\right|^{2}\right)^{1 / 2}\left(\sum_{i}\left|\mathcal{F}^{-1}\left(b_{i, j}^{k_{2}} \widehat{g}\right)(x)\right|^{2}\right)^{1 / 2} .
$$

Next we apply Hölder's inequality to obtain

$$
\begin{aligned}
& \left\|\mathfrak{M}^{2}(f, g)\right\|_{p} \\
& \leq \sum_{k_{1}, k_{2}} c^{k_{1}, k_{2}}\left\|\left(\sum_{i} \sup _{j}\left|\mathcal{F}^{-1}\left(a_{i, j}^{k_{1}} \widehat{f}\right)\right|^{2}\right)^{1 / 2}\right\|_{p_{1}}\left\|\left(\sum_{i} \sup _{j}\left|\mathcal{F}^{-1}\left(b_{i, j}^{k_{2}} \widehat{g}\right)\right|^{2}\right)^{1 / 2}\right\|_{p_{2}} .
\end{aligned}
$$

To finish this part of the proof, we observe that

$$
\begin{aligned}
& \sup _{j}\left|\mathcal{F}^{-1}\left(a_{i, j}^{k_{1}} \widehat{f}\right)\right| \leq C\left(1+\left|k_{1}\right|\right)^{d+1} M\left(\sum_{l=-n}^{n} \Delta_{i+l} f\right), \quad \text { and } \\
& \sup _{j}\left|\mathcal{F}^{-1}\left(b_{i, j}^{k_{2}} \widehat{g}\right)\right| \leq C\left(1+\left|k_{2}\right|\right)^{d+1} M\left(\sum_{l=-n}^{n} \Delta_{i+l} g\right),
\end{aligned}
$$

where $n$ is a fixed integer constant chosen so that $\sum_{l=-n}^{n} \phi\left(2^{-i+l}|\xi|\right)$ is equal to one on the support of the functions $a_{i, j}^{k_{1}}$ and $b_{i, j}^{k_{2}}$. To conclude the proof we first apply the Fefferman-Stein vector valued maximal function theorem (see [7]), then the Littlewood-Paley theorem, and we finally use the rapid decay of the $c^{k_{1}, k_{2}}$.

\section{Square function lemma}

To deal with $\mathfrak{M}^{1}$, first in the case $p>1$, we are going to combine the approaches from [5] and [9]. We decompose the symbol $m_{j}^{1}$ into dyadic pieces; denote $m_{j, i}^{1}=$ $\phi\left(2^{-i}|\cdot|\right) m_{j}^{1}$. Then the support of $m_{j, i}^{1}$ is contained in an $\epsilon$-interior of the product of some cubes $Q_{1}$ and $Q_{2}$ such that for any $\xi_{1} \in Q_{1}$ we have $C_{1}^{-1} 2^{i} \leq\left|\xi_{1}\right| \leq C_{1} 2^{i}$, while for any $\xi_{2} \in Q_{2}$ we have $\left|\xi_{2}\right| \leq C_{2} 2^{i}$ and, in particular, for large enough constant $K$, which we have used when decomposing the symbol, $C_{3}^{-1} 2^{i} \leq\left|\xi_{1}+\xi_{2}\right| \leq C_{3} 2^{i}$. (Here $C_{2}<<C_{1}^{-1}$.)

We now decompose each of the pieces $m_{j, i}^{1}$ using Lemma 2.1

$$
m_{j, i}^{1}\left(\xi_{1}, \xi_{2}\right)=\sum_{k_{1}, k_{2}} c_{i, j}^{k_{1}, k_{2}} a_{i, j}^{k_{1}}\left(\xi_{1}\right) b_{i, j}^{k_{2}}\left(\xi_{2}\right)
$$

where the function $a_{i, j}^{k_{1}}$ is supported in $Q_{1}$ and $b_{i, j}^{k_{2}}$ in $Q_{2}$ and $c_{i, j}^{k_{1}, k_{2}}$ have fast decay in $k_{1}, k_{2}$. It is obvious that the operator

$$
T_{m_{j, i}^{1}}(f, g)=\sum_{k_{1}, k_{2}} c^{k_{1}, k_{2}} \mathcal{F}^{-1}\left(\left(a_{i, j}^{k_{1}} \widehat{f}\right) *\left(b_{i, j}^{k_{2}} \widehat{g}\right)\right)
$$


gives a function whose Fourier transform is supported in $Q_{1}+Q_{2}$.

We need to establish a connection between localization of the Fourier image of a function and size of the dyadic martingale difference. Such estimates are fairly standard. Here we use the same lemma as in [9]. Let us first define two convolution operators.

We take a radial Schwartz function $b$ supported in $B(0,1 / 4)$, with $\widehat{b}(\xi) \neq 0$ for $C_{3}^{-1} \leq|\xi| \leq C_{3}$ and $\int_{\mathbb{R}^{d}} b(\xi) \mathrm{d} \xi=0$ and put $\widehat{b}_{i}(\xi)=\widehat{b}\left(2^{-i} \xi\right)$. Pick a Schwartz function $\iota$ such that $\widehat{\iota}$ is supported in $\left\{\xi:|\xi| \in\left[C_{3}^{-1} / 3,3 C_{3}\right]\right\}$ such that for $\xi \in\left[C_{3}^{-1} / 2, C_{3} / 2\right]$ we have $\widehat{\iota}(\xi)(\widehat{b}(\xi))^{2}=1$. We shall denote

$$
\begin{aligned}
& B_{i} f=f * b_{i} \\
& I_{i} f=f * \iota_{i} .
\end{aligned}
$$

Lemma 6.1. For $i, k \in \mathbb{Z}$ and $s>1$ we have

$$
\left|\left(B_{i} I_{i} f\right)(\xi)\right| \leq C M f(\xi)
$$

and

$$
\left|\left(\mathbb{D}_{k} B_{i+k} f\right)(\xi)\right| \leq C 2^{-|i| / s^{\prime}} M_{s} f(\xi) .
$$

Proof. As inequality (6.1) is trivial, we sketch the proof of (6.2). In the case $i>0$ the stronger estimate

$$
\left|\left(\mathbb{D}_{k} B_{i+k} f\right)(\xi)\right| \leq C 2^{i} M f(\xi)
$$

follows easily from the smoothness of $b$ using cancelation.

In the case $i<0$ we estimate the quantity $\left|\left(\mathbb{E}_{k} B_{i+k} f\right)(\xi)\right|$. Pick the cube $Q \in \mathcal{D}_{k}$ with $\xi \in Q$. Denote

$$
Q^{1}=\left\{x: \operatorname{dist}(x, \partial Q) \leq 2^{-k+i}\right\} .
$$

Since $b$ has mean value 0 we have

$$
\mathbb{E}_{k} B_{i+k} f(\xi)=\left(\mathbb{E}_{k} B_{i+k} f \chi_{Q^{1}}\right)(\xi) .
$$

Denote

$$
Q^{2}=\left\{x: \operatorname{dist}\left(x, Q^{1}\right) \leq 2^{-k+i}\right\},
$$

clearly $B_{i+k} f \chi_{Q^{1}}$ is supported inside $Q^{2}$. The measure of the set $Q^{2}$ is comparable to $2^{-k d+i}$ and (6.2) then follows by an application of Hölder's inequality to

$$
\mathbb{E}_{k} B_{i+k} f(\xi)=\left(\mathbb{E}_{k}\left(\chi_{Q^{2}} B_{i+k} f \chi_{Q^{1}}\right)\right)(\xi) .
$$

We now define the auxiliary operator

$$
F_{s}(f, g)(x)=\left(\sum_{k \in \mathbb{Z}}\left(M M\left(M\left|\left(\Delta_{k-1}+\Delta_{k}+\Delta_{k+1}\right) f\right|\right)^{s} M g\right)^{2 / s}\right)^{1 / 2}
$$

and we prove the following lemma concerning it.

Lemma 6.2. For any $1<s<\infty$ and for $1 \leq j \leq N$ we have a pointwise estimate

$$
S\left(T_{m_{j}^{1}}(f, g)\right)(x) \leq C_{s} F_{s}(f, g)(x) .
$$


Proof. Let us write

$$
\left|\mathbb{D}_{k} T_{m_{j}^{1}}(f, g)\right|(x) \leq \sum_{n \in \mathbb{Z}, l \in \mathbb{Z}^{2}} c^{l}\left|\left(\mathbb{D}_{k} B_{k+n}\right)\left(B_{k+n} I_{k+n}\right) \mathcal{F}^{-1}\left(\left(a_{n+k, j}^{l_{1}} \widehat{f}\right) *\left(b_{n+k, j}^{l_{2}} \widehat{g}\right)\right)\right| .
$$

Again, we have $c^{l}=\sup c_{i, j}^{l}$ is a sequence with decay faster that $C_{K}|l|^{-K}$ for any $K \in \mathbb{N}$. Note that

$$
\begin{aligned}
& \left|\mathcal{F}^{-1}\left(\left(a_{n+k, j}^{l_{1}} \widehat{f}\right) *\left(b_{n+k, j}^{l_{1}} \widehat{g}\right)\right)\right| \\
& =\left|\mathcal{F}^{-1}\left(a_{n+k, j}^{l_{1}} \widehat{f}\right) \mathcal{F}^{-1}\left(b_{n+k, j}^{l_{1}} \widehat{g}\right)\right| \\
& \leq(1+|l|)^{2 d} C\left(M\left|\left(\Delta_{n+k-1}+\Delta_{n+k}+\Delta_{n+k+1}\right) f\right|\right)(M g) .
\end{aligned}
$$

Next we use estimates (6.2) and (6.1) to obtain

$$
\begin{aligned}
& \left|\mathbb{D}_{k} T_{m_{j}^{1}}(f, g)(x)\right| \\
& \leq C \sum_{n \in \mathbb{Z}, l \in \mathbb{Z}^{2}} 2^{-|n+k| / s^{\prime}}|l|^{-K}(1+|l|)^{2 d} M_{s} M\left(\left(M\left|\left(\Delta_{n+k-1}+\Delta_{n+k}+\Delta_{n+k+1}\right) f\right|\right)(M g)\right) .
\end{aligned}
$$

At this point we can choose $K$ so large that we eliminate the variable $l$ and after we pass to the square function, we can drop the dependence on $n$. We have the estimate

$$
S\left(T_{m_{j}^{1}}(f, g)\right)(x) \leq C\left(\sum_{k}\left(M_{s} M\left(\left(M\left|\left(\Delta_{k-1}+\Delta_{k}+\Delta_{k+1}\right) f\right|\right)(M g)\right)\right)^{2}\right)^{1 / 2},
$$

and the result follows.

Let us establish the boundedness of the operator $F_{s}$.

Lemma 6.3. Assume that $1<s<\min \{p, 2\}$. Then we have

$$
\left\|F_{s}(f, g)\right\|_{p} \leq C\|f\|_{p_{1}}\|g\|_{p_{2}} .
$$

Proof. We prove this by repeated application of the Fefferman-Stein vector valued maximal function result [7]. The Littlewood-Paley theory gives that

$$
\left\|\left(\sum_{k}\left|\left(\Delta_{k-1}+\Delta_{k}+\Delta_{k+1}\right) f\right|^{2}\right)^{1 / 2}\right\|_{p_{1}} \leq C\|f\|_{p_{1}} .
$$

Applying the vector valued maximal function and Hölder's inequalities, we obtain

$$
\left\|\left(\sum_{k}\left(M\left|\left(\Delta_{k-1}+\Delta_{k}+\Delta_{k+1}\right) f\right|(M g)\right)^{2}\right)^{1 / 2}\right\|_{p} \leq C\|f\|_{p_{1}}\|g\|_{p_{2}} .
$$

The result then follows by using Fefferman-Stein inequality twice in the space $L^{p / s}\left(l^{2 / s}\right)$. 


\section{The part $\mathfrak{M}^{1}$}

Now we are ready to apply the method of [9] to the operator $\mathfrak{M}^{1}$. We shall first prove the result in the case $p>1$. We are going to pick $1<s<\min \{p, 2\}$. The key idea is to apply the good- $\lambda$ inequality (Proposition 3.1) from [3], which states that

$$
\left|\left\{\sup _{k}\left|\mathbb{E}_{k} f-\mathbb{E}_{0} f\right|>2 \lambda\right\} \cap\{S(f)<\epsilon \lambda\}\right| \leq C e^{C_{d} / \epsilon^{2}}\left|\left\{\sup _{k}\left|\mathbb{E}_{k} f\right|>\lambda\right\}\right|
$$

for any $\lambda>0$ and $1>\epsilon>0$. The inequality is stated for a martingale inside unit cube, but it is clear that it can be extended to one-sided martingale on $\mathbb{R}^{d}$ starting at level $k_{0}$. We need to treat the initial expectation separately. We can assume that the Fourier transform of $f$ is supported away from origin. By previous considerations $\mathcal{F}\left(T_{m_{j}^{1}}(f, g)\right)$ is then supported outside some ball $B_{\left(0,2^{\delta}\right)}$. Using 6.2 we can estimate

$$
\left|\mathbb{E}_{0} T_{m_{j}^{1}}(f, g)(x)\right|=\left|\sum_{i=\delta-1}^{\infty} \mathbb{E}_{0} \Delta_{i} T_{m_{j}^{1}}(f, g)(x)\right| \leq C 2^{-k_{0}+\delta / s^{\prime}}\left|M_{s} T_{m_{j}^{1}}(f, g)(x)\right| .
$$

Now we can select the starting level $k_{0}$, based on the value of $\delta$, such that

$$
\left\|\mathbb{E}_{0} T_{m_{j}^{1}}(f, g)\right\|_{p} \leq N^{-1}\left\|T_{m_{j}^{1}}(f, g)\right\|_{p} .
$$

We have

$$
\left\|\mathfrak{M}^{1}(f, g)\right\|_{p}^{p}=p 4^{p} \int_{0}^{\infty} \lambda^{p-1}\left|\left\{\mathfrak{M}^{1}(f, g)>4 \lambda\right\}\right| \mathrm{d} \lambda
$$

Of course

$$
\begin{aligned}
& \left|\left\{\mathfrak{M}^{1}(f, g)>4 \lambda\right\}\right| \\
& \leq \sum_{j}\left|\left\{\left|T_{m_{j}^{1}}(f, g)-\mathbb{E}_{0} T_{m_{j}^{1}}(f, g)\right|>2 \lambda\right\} \cap\left\{F_{s}(f, g)<\epsilon \lambda\right\}\right| \\
& \quad+\left|\left\{F_{s}(f, g) \geq \epsilon \lambda\right\}\right|+\sum_{j}\left|\left\{\left|\mathbb{E}_{0} T_{m_{j}^{1}}(f, g)\right|>2 \lambda\right\}\right| \\
& \leq \mid\left\{\left(F_{s}(f, g) \geq \epsilon \lambda\right\}\left|+\sum_{j}\right|\left\{\left|T_{m_{j}^{1}}(f, g)\right|>2 \lambda\right\} \cap\left\{S\left(T_{m_{j}^{1}}(f, g)\right)<C \epsilon \lambda\right\} \mid\right. \\
& \quad+\sum_{j}\left|\left\{\left|\mathbb{E}_{0} T_{m_{j}^{1}}(f, g)\right|>2 \lambda\right\}\right| .
\end{aligned}
$$

Now we can apply 7.1 to each term of the first sum with $\epsilon=(\log N)^{-1 / 2} / C_{s}$. After integration, this gives the norm estimate

$$
\begin{aligned}
\left\|\mathfrak{M}^{1}(f, g)\right\|_{p} \leq C & (\log N)^{1 / 2}\left\|F_{s}(f, g)\right\|_{p}+\frac{C}{N} \sum_{j \leq N}\left\|M T_{m_{j}^{1}}(f, g)\right\|_{p} \\
& +C \sum_{j}\left\|\mathbb{E}_{0} T_{m_{j}^{1}}(f, g)\right\|_{p} .
\end{aligned}
$$

Now the norm of each of the terms $\left\|M T_{m_{j}^{1}}(f, g)\right\|_{p}$ is uniformly controlled, while $\left\|F_{s}(f, g)\right\|_{p}$ is bounded according to Lemma 6.3. In view of (7.2) we can assume that each of the terms $\left\|\mathbb{E}_{0} T_{m_{j}^{1}}(f, g)\right\|_{p}$ is bounded by $N^{-1}\left\|T_{m_{j}^{1}}(f, g)\right\|_{p}$. This finishes the proof for the part $\mathfrak{M}^{1}$ provided $p>1$. 


\section{Weak type estimate}

So far, we have proved Theorem 1.1 in the case $p>1$. To extend the result to $p>1 / 2$ we are going to use an endpoint weak type estimate. The linear case of this theorem was proved in [6], see also [4]. To prove the bilinear version we are going to use an argument adapted from [10]. Similar aproach can be of course used to obtain general multilinear version.

Theorem 8.1. Let us have a countable family of bilinear multipliers $\left\{m_{j}\right\}$ such that the norm of the associated maximal operator $\mathfrak{M}$ is bounded from $L^{p_{1}}\left(\mathbb{R}^{d}\right) \times L^{p_{2}}\left(\mathbb{R}^{d}\right)$ into $L^{p}\left(\mathbb{R}^{d}\right)$ by a constant $A$ for some $p_{i}$ and $p$ as in (1.2). Let us assume that the condition (1.1) is satisfied uniformly for all $m_{j}$. Then the operator $\mathfrak{M}$ is bounded from $L^{1} \times L^{1}$ into $L^{1 / 2, \infty}$ with the norm at most $C_{d}(A+B)$, where $B$ is a constant dependent only on the constants from the condition (1.1).

Once this theorem is proved, the use standard multilinear interpolation argument finishes the proof of the Theorem 1.1.

Proof. We are going to fix Schwartz functions $f_{1}$ and $f_{2}$ such that $\left\|f_{1}\right\|_{1}=\left\|f_{2}\right\|_{1}=1$. To prove the theorem, we need to show that for any $\alpha>0$ we have

$$
|E|^{2} \leq C(A+B) \alpha^{-1},
$$

where $E=\left\{\mathfrak{M}\left(f_{1}, f_{2}\right)>\alpha\right\}$.

It is well known that if we define distributional kernels

$$
K_{j}=\mathcal{F}^{-1}\left(m_{j}\right),
$$

we can write

$$
T_{m_{j}}\left(f_{1}, f_{2}\right)(x)=\int K_{j}(x-y, x-z) f_{1}(y) f_{2}(z) d y d z
$$

in the sense of distributions. The distribution $K$ is a function away from the origin. Standard argument shows that the following estimate holds

$$
\left|\partial^{\beta} K_{j}\left(x_{1}, x_{2}\right)\right| \leq C\left(\left|x_{1}\right|+\left|x_{2}\right|\right)^{-(2 d+|\beta|)}
$$

for any $j$ and multiindex $|\beta| \leq 1$.

Now we shall perform the Calderón-Zygmund decomposition at the level $(\alpha \gamma)^{1 / 2}$ where $\gamma=A^{-1}$, let us put for both of the functions $f_{i}=b_{i}+g_{i}$. Let us remind that for the function $g_{i}$ we have

$$
\left\|g_{i}\right\|_{s} \leq C(\alpha \gamma)^{1 / 2 s^{\prime}}
$$

for every $s \in[1, \infty]$. The function $b_{i}$ can be written as $b_{i}=\sum_{l} b_{i, l}$, where each of the functions $b_{i, l}$ is supported in a cube $Q_{i, l}$ with center $c_{i, l_{i}}$ and the cubes with same index $i$ have disjoint interiors. Furthermore, we have

$$
\begin{gathered}
\int b_{i, l}(x) \mathrm{d} x=0, \\
\int\left|b_{i, l}(x)\right| \mathrm{d} x \leq C(\alpha \gamma)^{1 / 2}\left|Q_{i, l}\right|, \\
\left|\cup_{l} Q_{i, l}\right| \leq C(\alpha \gamma)^{1 / 2}
\end{gathered}
$$

and

$$
\left\|b_{i}\right\|_{1} \leq C
$$


It is clear that any $x \in E$ belongs to a set of the form $\left\{\mathfrak{M}\left(h_{1}, h_{2}\right)>\alpha / 4\right\}$ where each $h_{i} \in\left\{g_{i}, b_{i}\right\}$. Thus, we need to prove (8.1) for all four combination of $h_{i}$ 's.

First, the $\left(p_{1}, p_{2}, p\right)$ boundedness of $\mathcal{M}$ gives by the Chebychev's inequality

$$
\begin{aligned}
\left|\left\{\mathfrak{M}\left(g_{1}, g_{2}\right)>\alpha / 4\right\}\right| & \leq C(A / \alpha)^{p}\left\|g_{1}\right\|_{p_{1}}^{p}\left\|g_{2}\right\|_{p_{2}}^{p} \\
& \leq C A^{p} \alpha^{-1 / 2} \gamma^{p-1 / 2}=C A^{1 / 2} \alpha^{-1 / 2} .
\end{aligned}
$$

Next, we need to estimate the terms which contains $o$ functions $b_{i}, o \in\{1,2\}$. To simplify the notation, we shall only prove that

$$
\begin{gathered}
\left|\left\{\mathfrak{M}\left(b_{1}, g_{2}\right)>\alpha / 4\right\}\right| \leq C(A+B)^{1 / 2} \text { and } \\
\left|\left\{\mathfrak{M}\left(b_{1}, b_{2}\right)>\alpha / 4\right\}\right| \leq C(A+B)^{1 / 2} .
\end{gathered}
$$

Let us now take a point $x$ outside $\cup_{i, l} 2 Q_{i, l}$. The sublinearity gives

$$
\begin{aligned}
& \mathfrak{M}\left(b_{1}, g_{2}\right)(x) \leq \sum_{l_{1}} \mathfrak{M}\left(b_{1, l_{1}}, g_{2}\right)(x) \quad \text { and } \\
& \mathfrak{M}\left(b_{1}, b_{2}\right)(x) \leq \sum_{l_{1}, l_{2}} \mathfrak{M}\left(b_{1, l_{1}}, b_{2, l_{2}}\right)(x) .
\end{aligned}
$$

Let us fix indices $l_{1}, l_{2}$ and assume that the cube $Q_{1, l_{1}}$ is smaller than $Q_{2, l_{2}}$. The smoothess of $K_{j}$ gives in the usual way

$$
\left|\int_{Q_{1, l_{1}}} K_{j}\left(x-y_{1}, x-y_{2}\right) b_{1, l_{1}}\left(y_{1}\right) \mathrm{d} y_{1}\right| \leq C B \int_{Q_{1, l_{1}}} \frac{\left|b_{1, l_{1}}\left(y_{1}\right)\right| \operatorname{diam}\left(Q_{1, l_{1}}\right)}{\left(\left|x-y_{1}\right|+\left|x-y_{2}\right|\right)^{2 d+1}} \mathrm{~d} y_{1} .
$$

Thus we have either

$$
\begin{aligned}
& \int_{\mathbb{R}^{2 d}} b_{1, l_{1}}\left(y_{1}\right) b_{2, l_{2}}\left(y_{2}\right) K_{j}\left(x-y_{1}, x-y_{2}\right) \mathrm{d} y_{1} \mathrm{~d} y_{2} \\
& \leq C B \int_{Q_{2, l_{2}}} b_{2, l_{2}}\left(y_{2}\right) \int_{Q_{1, l_{1}}} \frac{\left|b_{1, l_{1}}\left(y_{1}\right)\right| \operatorname{diam}\left(Q_{1, l_{1}}\right)}{\left(\left|x-y_{1}\right|+\left|x-y_{2}\right|\right)^{2 d+1}} \mathrm{~d} y_{1} \mathrm{~d} y_{2} \\
& \leq C B\left\|b_{1, l_{1}}\right\|_{1}\left\|b_{2, l_{2}}\right\|_{1} \frac{\operatorname{diam}\left(Q_{1, l_{1}}\right)}{\left(\operatorname{diam}\left(Q_{1, l_{1}}\right)+\left|x-c_{1, l_{1}}\right|+\operatorname{diam}\left(Q_{2, l_{2}}\right)+\left|x-c_{2, l_{2}}\right|\right)^{2 d+1}}
\end{aligned}
$$

or

$$
\begin{aligned}
& \int_{\mathbb{R}^{2 d}} b_{1, l_{1}}\left(y_{1}\right) g_{2}\left(y_{2}\right) K_{j}\left(x-y_{1}, x-y_{2}\right) \mathrm{d} y_{1} \mathrm{~d} y_{2} \\
& \leq C B\left\|b_{1, l_{1}}\right\|_{1}\left\|g_{2}\right\|_{\infty} \frac{\operatorname{diam}\left(Q_{1, l_{1}}\right)}{\left(\operatorname{diam}\left(Q_{1, l_{1}}\right)+\left|x-c_{1, l_{1}}\right|\right)^{d+1}}
\end{aligned}
$$

The cube $Q_{1, l_{1}}$ is the smaller one, so we can write

$$
\begin{aligned}
& \frac{\operatorname{diam}\left(Q_{1, l_{1}}\right)}{\left(\operatorname{diam}\left(Q_{1, l_{1}}\right)+\left|x-c_{1, l_{1}}\right|+\operatorname{diam}\left(Q_{2, l_{2}}\right)+\left|x-c_{2, l_{2}}\right|\right)^{2 d+1}} \\
& \leq C \prod_{i=1}^{2} \frac{\operatorname{diam}\left(Q_{i, l_{i}}\right)^{1 / 2}}{\left.\left(\operatorname{diam}\left(Q_{i, l_{i}}\right)+\left|x-c_{i, l_{i}}\right|\right)\right)^{d+1 / 2}}
\end{aligned}
$$

Since these estimates do not depend on $j$ we have

$$
\mathfrak{M}\left(b_{1}, g_{2}\right)(x) \leq C B(\alpha \gamma)^{1 / 2} \sum_{l_{1}} \frac{\left\|b_{1, l_{1}}\right\|_{1} \operatorname{diam}\left(Q_{i, l_{i}}\right)^{1 / 2}}{\left.\left(\operatorname{diam}\left(Q_{i, l_{i}}\right)+\left|x-c_{i, l_{i}}\right|\right)\right)^{d+1 / 2}}
$$


and

$$
\mathfrak{M}\left(b_{1}, b_{2}\right)(x) \leq C B \prod_{i=1}^{2}\left(\sum_{l_{1}, l_{2}} \frac{\left\|b_{i, l_{i}}\right\|_{1} \operatorname{diam}\left(Q_{i, l_{i}}\right)^{1 / 2}}{\left.\left(\operatorname{diam}\left(Q_{i, l_{i}}\right)+\left|x-c_{i, l_{i}}\right|\right)\right)^{d+1 / 2}}\right)
$$

The sum in the last product is called Marcinkiewicz fuction, and a well known estimate (see [14]) says that

$$
\int_{\mathbb{R}^{d}} \sum_{k_{i}} \frac{\operatorname{diam}\left(Q_{i, k_{i}}\right)^{d+1 / 2}}{\left.\left(\operatorname{diam}\left(Q_{i, l_{i}}\right)+\left|x-c_{i, l_{i}}\right|\right)\right)^{d+1 / 2}} \mathrm{~d} x \leq C\left|\cup_{k_{i}} Q_{i, k_{i}}\right| \leq C(\alpha \gamma)^{-1 / 2} .
$$

The estimate (8.1) then follows the usual way, the size of the set $\cup_{i, l} 2 Q_{i, l}$ is controlled by $C(\alpha \gamma)^{-1 / 2}$ and outside we use the just derived estimate and Hölder's inequality.

\section{Acknowledgements}

Honzík was supported by the Institutional Research Plan no. AV0Z10190503 of the Academy of Sciences of the Czech Republic (AS CR) and by the grant KJB100190901 GAAV

\section{References}

[1] D.L. Burkholder, Distribution function inequalities for martingales, Ann. Prob. 1 (1973), $19-42$.

[2] D.L. Burkholder, B. Davis and R. Gundy, Integral inequalities for convex functions of operators on martingales, Proc. Sixth Berkeley Symp. Math. Statist. Prob. 2 (1972), 223-240.

[3] S.Y.A. Chang, M. Wilson and T. Wolff, Some weighted norm inequalities concerning the Schrödinger operator, Comment. Math. Helv. 60 (1985), 217-246.

[4] M. Christ, L. Grafakos, P. Honzík and A. Seeger, Maximal functions associated with multipliers of Mikhlin-Hörmander type, Math. Zeit. 249 (2005), 223-240.

[5] R. Coifman and Y. Meyer, Commutateurs d'intégrales singuliéres et opérateurs multilinéaires, Ann. Inst. Fourier (Grenoble) 28 (1978), 177-202.

[6] H. Dappa and W. Trebels, On maximal functions generated by Fourier multipliers, Ark. Mat. 23 (1985), 241-259.

[7] C. Fefferman and E. M. Stein, Some maximal inequalities, Amer. J. Math. 93 (1971), 107-115.

[8] L. Grafakos, Classical and Modern Fourier Analysis, Prentice Hall, Pearson Education, Upper Saddle River, NJ, 2003.

[9] L. Grafakos, P. Honzík, and A. Seeger, Sharp estimates for maximal functions associated with multipliers of Mikhlin-Hörmander type, submitted.

[10] L. Grafakos and R. Torres, Multilinear Calderón-Zygmund theory, Adv. Math. 165 (2002), $124-164$.

[11] L. Hörmander, Estimates for translation invariant operators in $L^{p}$ spaces, Acta Math. 104 (1960), 93-139.

[12] C. E. Kenig and E. M. Stein, Multilinear estimates and fractional integration, Math. Res. Lett. 6 (1999), 1-15.

[13] S. G. Mikhlin, On the multipliers of Fourier integrals (Russian) Dokl. Akad. Nauk SSSR (N.S.) 109 (1956), 701-703.

[14] E. M. Stein, Singular Integrals and Differentiability Properties of Functions, Princeton Univ. Press, Princeton, NJ, 1971.

Petr Honzík, Institute of Mathematics, AS CR, Žitná 25, CZ - 11567 Praha 1, Czech REPUBLIC

E-mail address: honzikp@gmail.com 